\title{
Validação de tecnologias educacionais na área da saúde: protocolo de scoping review
}

\author{
Validation of educational technologies in health care: scoping review protocol \\ Validación de las tecnologías educativas en la atención sanitaria: protocolo de revisión del alcance
}

Recebido: 03/12/2021 | Revisado: 09/12/2021 | Aceito: 13/12/2021 | Publicado: 21/12/2021

\author{
Alexandy Michel Dantas Santos \\ ORCID: https://orcid.org/0000-0003-0905-8236 \\ Universidade Federal do Rio Grande do Norte, Brasil \\ E-mail: michelsantos1993@gmail.com \\ Ezequiel Benigno Resende \\ ORCID: https://orcid.org/0000-0001-6350-5100 \\ Universidade Federal do Rio Grande do Norte, Brasil \\ E-mail: ezequiel.b.resende@ hotmail.com \\ Cláudia Cristiane Filgueira Martins Rodrigues \\ ORCID: https://orcid.org/0000-0001-5182-2491 \\ Universidade Federal do Rio Grande do Norte, Brasil \\ E-mail: claudiacrisfm@yahoo.com.br \\ Kisna Yasmin Andrade Alves \\ ORCID: https://orcid.org/0000-0001-7900-0262 \\ Universidade Federal do Rio Grande do Norte, Brasil \\ E-mail: kisnayasmin@ hotmail.com \\ Lannuzya Veríssimo e Oliveira \\ ORCID: https://orcid.org/0000-0002-6881-898X \\ Universidade Federal do Rio Grande do Norte, Brasil \\ E-mail: lannuzyacg@hotmail.com \\ Pétala Tuani Candido de Oliveira Salvador \\ ORCID: https://orcid.org/0000-0002-3208-6270 \\ Universidade Federal do Rio Grande do Norte, Brasil \\ E-mail: petalatuani@hotmail.com
}

\begin{abstract}
Resumo
Este artigo apresenta o protocolo de revisão de escopo com o objetivo de mapear o processo de validação das tecnologias educacionais na área da saúde. Esta revisão será conduzida seguindo as diretrizes do Joanna Briggs Institute (JBI) e será previamente registrada no repositório Open Science Framework. Guiada pela questão de pesquisa: Como tem ocorrido o processo de validação de tecnologias educacionais na área da saúde? A busca será realizada em oito bases de dados. Será utilizado o software Rayyan para gerenciamento da coleta e seleção dos estudos. Com base nos critérios de inclusão e exclusão estabelecidos, será realizada a leitura do título e resumo de todos os estudos identificados por dois revisores de forma independente e um terceiro revisor para resolver possíveis divergências. Os dados serão extraídos, sintetizados e apresentados de forma descritiva, quadros e/ou imagens, conforme indicado no checklist proposto pelo PRISMA-ScR. Espera-se que este protocolo possibilite a realização de uma revisão de escopo com metodologia consistente e, a partir dela, a obtenção de maiores conhecimentos e orientação quanto ao processo de validação de tecnologias educacionais na área da saúde.
\end{abstract}

Palavras-chave: Estudo de validação; Ciências da saúde; Tecnologia educacional.

\begin{abstract}
This manuscript presents the scope review protocol which aims to map the validation process of educational technologies in the health field. This review wiil be conducted according to Joanna Briggs Institute (JBI) guidelines and will be previously registered in the Open Science Framework repository. Guided by the survey question: How has validation process of educational technologies in the health field been taking place? The survey will occur in eight database. It will be utilized the Rayyan Software for managing selection and collection of studies. Based on inclusion and exclusion established criteria, two independent reviewers will perform the title and abstract reading of all identified studies. In case of divergences between the reviewers, a third reviewer will be requested. The data will be extracted, summarized and descriptively presented, making use of frames and images, as indicated in the checklist proposed by PRISMA-ScR. We expect this protocol enables a scope review with a consistent methodology and from it, greater knowledge and guidance concerning validation process of educational technologies in the health field.
\end{abstract}

Keywords: Validation study; Health sciences; Educational technology.

\section{Resumen}

Este documento presenta el protocolo de revisión del alcance con el objetivo de mapear el proceso de validación de las tecnologías educativas en el ámbito de la salud. Esta revisión se realizará siguiendo las directrices del Instituto 
Joanna Briggs (JBI) y se registrará previamente en el repositorio Open Science Framework. Guiados por la pregunta de investigación: ¿Cómo se ha producido el proceso de validación de las tecnologías educativas en la salud? La búsqueda se realizará en ocho bases de datos. El programa informático Rayyan se utilizará para gestionar la recogida y selección de estudios. En base a los criterios de inclusión y exclusión establecidos, el título y el resumen de todos los estudios identificados serán leídos por dos revisores de forma independiente y un tercer revisor para resolver las posibles diferencias. Los datos se extraerán, sintetizarán y presentarán en forma descriptiva, tablas y/o imágenes, como se indica en la lista de comprobación propuesta por PRISMA-ScR. Se espera que este protocolo permita la realización de una revisión del alcance con una metodología consistente y, a partir de ella, la obtención de un mayor conocimiento y orientación sobre el proceso de validación de las tecnologías educativas en el área de la salud.

Palabras clave: Estudio de validación; Health sciences; Tecnología educacional.

\section{Introdução}

Cercado por múltiplos conceitos, a definição de tecnologia faz alusão a um produto ou processo produtivo resultado da atividade humana, que permite a reestruturação da vida dos indivíduos ao promover o desenvolvimento de técnicas com instrumentos físicos, materiais ou midiáticos, sendo capaz de proporcionar uma mudança de comportamento ao ser associada a outros aspectos da vida do sujeito (Nespoli, 2013; Paim, Nietsche \& Lima, 2014). O desenvolvimento tecnológico, portanto, insere-se em um contexto de mudanças e inovação em resposta a alguma demanda específica. Nesse cenário, destaca-se o contexto educacional, em especial o ensino na área da saúde.

Buscando atualizar-se juntamente com as crescentes necessidades de dinamização no processo do ensino, os profissionais de saúde vêm implementando diversas ferramentas que favorecem o processo de cuidar e educar. Assim, frente à demanda por metodologias de ensino mais dinâmicas e à necessidade de desenvolvimento de competências profissionais pautadas no protagonismo, criticidade e criatividade, as Tecnologias Educacionais (TE) se apresentam como práticas sociotécnicas que mediam o processo de ensinar e aprender de modo a torná-lo mais ativo e colaborativo, podendo ser utilizadas em processos coletivos de construção do conhecimento e em todos os níveis de ensino, seja ele acadêmico ou durante a prática assistencial (Paim et al., 2014; Teixeira, 2010).

Necessário destacar, no âmbito desta discussão, que toda a sociedade sofre atualmente com os transtornos vivenciados em virtude da pandemia de COVID-19, causada pelo agente etiológico SARS-CoV-2 (Severe Acute Respiratory Syndrome Coronavirus) (Harapan et al., 2020), o que também trouxe importantes impactos para os ambientes de ensino. Assim como em vários países, o Brasil adotou medidas que objetivou a redução da contaminação por COVID-19, como o distanciamento social, higienização das mãos com frequência, uso de máscara, etiqueta respiratória e substituição de aulas presenciais por aulas mediadas por TE, a fim de possibilitar a continuidade do ensino (Brasil, 2021, 2020). Neste contexto, as TE se apresentam como forma adaptativa e eficiente no processo de educação, no qual o processo de troca presencial de saberes se encontra impossibilitada.

Enfatiza-se, todavia, a compreensão de que o ensino mediado por tecnologias não é próprio da modalidade a distância ou remota, termo mais utilizado hodiernamente. Compreende-se que o uso de TE nos contextos educacionais é imperativo em todas as modalidades de ensino e constitui uma demanda para a formação de competências profissionais para as quais o ensino tradicional é insuficiente.

No que diz respeito ao contexto da saúde, seus profissionais se apresentam também como educadores e, para isto, utilizam diversas ferramentas que favorecem o processo de cuidar e educar. Ao valer-se de uma TE como uma ferramenta de ensino, é proporcionado o aprimoramento do cuidado em saúde e uma participação ativa dos sujeitos envolvidos no processo educacional (Nascimento, Silva, Oliveira, Nascimento, \& Ferreira, 2020; Nietsche et al., 2012; Santos, Lopes, Passos \& Néri, 2020).

Ressalta-se, ainda, que o uso de TE no processo de ensino-aprendizagem possibilita a aquisição de novos conhecimentos, em que o uso destas ferramentas proporciona o acesso a um conteúdo educacional fundamentado em 
evidências científicas, ativo e de fácil compreensão, favorecendo a melhoria da saúde do sujeito assistido e pondo em prática atitudes de autocuidado e prevenção (Nietsche et al., 2012).

Destarte, elucida-se que a TE pode ser utilizada em todas as áreas de atuação do profissional da saúde, sendo ele educador ou assistencial, de modo a estimular um ensino dialógico, proporcionando ao educando uma posição de protagonismo do seu aprendizado e favorecendo acesso a um conhecimento objetivo, de linguagem simples e visualmente atrativa (Rodrigues, Teixeira, \& Nogueira, 2019).

Apresentando-se como uma ferramenta de caráter complementar às consultas e palestras exercidas pelos educadores em saúde (Rodrigues et al., 2019), a TE propõe a solidificação do conhecimento passado, diminuição de dúvidas e apropriação das informações transmitidas pelos educadores (Paz \& Teixeira, 2021).

No entanto, é fundamental apreender que a validação da TE deve ocorrer antes que a mesma seja utilizada na prática assistencial ou nos ambientes acadêmicos, uma vez que realizar esta validação possibilita a fundamentação, precisão e confiabilidade dos dados apresentados, isso porque sujeitos especialistas na área da ferramenta realizam uma amostragem e análise criteriosa do conteúdo, podendo, ainda, valer-se de índices utilizados para calcular a relevância dos itens e concordância entre os especialistas (Polit \& Beck, 2011; Teixeira \& Mota, 2011), como o Índice de Validade do Conteúdo (ICV), concordância interobservadores e o coeficiente de kappa (Alexandre \& Coluci, 2011).

Destaca-se que a validação de uma TE objetiva verificar a pertinência do seu conteúdo e de outros aspectos, como aparência e usabilidade, a fim de tornar o instrumento confiável, replicável e com informações importantes sobre público alvo (Pasquali, 2010). Por este motivo, o processo de validação é de caráter indispensável no desenvolvimento de uma TE, visto que a ausência deste artifício por parte de alguns estudos que utilizam tecnologias ou programas educacionais no processo de educação em saúde e educação continuada pode refletir no comprometimento da qualidade da tecnologia, com implicações na efetividade de sua utilização para fins educacionais (Carvalho et al., 2020).

Ressalta-se que até o presente momento não foram identificados na literatura nacional e internacional estudos de revisão que abordem a validação de tecnologias educacionais no âmbito da saúde. Dessa maneira, a presente pesquisa poderá contribuir para o preenchimento dessa lacuna e seus resultados podem subsidiar a prática de profissionais de saúde ao permitir o conhecimento sobre a validação de TEs na saúde e, ao mesmo tempo, promover reflexões quanto ao uso destas tecnologias no processo de educação e identificar lacunas sobre esta temática. Desse modo, o presente estudo objetiva mapear o processo de validação das tecnologias educacionais na área da saúde.

\section{Metodologia}

Esta revisão do escopo será conduzida de acordo com a metodologia definida pelo manual for evidence synthesis de Joanna Briggs Institute (JBI) de agosto de 2020, seguindo o quadro referencial proposto (Peters et al., 2020). O protocolo foi elaborado e registrado no Open Science Framework (OSF) com o número de registro DOI 10.17605/OSF.IO/2YVKE a fim de garantir a transparência da revisão.

\subsection{Pergunta da revisão}

A pergunta norteadora desta revisão englobará elementos do acrônimo PCC (Participantes, Conceito, Contexto), onde orienta também a definição dos critérios de inclusão para a revisão de escopo. Desta forma, a pergunta norteadora desta revisão

é: Como tem ocorrido o processo de validação de tecnologias educacionais na área da saúde? 


\subsection{Critério de inclusão}

\subsubsection{População}

A população desta revisão será constituída por estudos acerca das tecnologias educacionais. Dessa maneira, TE é compreendida como um conjunto de práticas sociotécnicas que mediam o processo de ensinar e aprender, podendo ser utilizadas em construções coletivas do conhecimento e em todos os níveis de ensino, seja ele acadêmico ou durante a prática assistencial (Paim et al., 2014; Teixeira, 2010).

\subsubsection{Conceito}

Esta revisão considerará estudos que abordem processos de validação de tecnologias educacionais. Portanto, serão consideradas a forma e a referência para as quais se basearam a validação da tecnologia.

Diante disto, têm-se "validação" como uma investigação científica que busca a avaliação criteriosa de um comitê de especialistas da área na qual a tecnologia está sendo desenvolvida, em que estes deverão ajuizar os itens do instrumento e, por fim, o instrumento como um todo. Portanto, o processo de validação irá possibilitar a utilização de instrumentos confiáveis e apropriados para a população na qual a TE será empregada (Alexandre \& Coluci, 2011).

\subsubsection{Contexto}

Esta revisão considerará estudos que abordem processos de validação conduzidos em qualquer nível educacional da área da saúde, podendo ser no processo de educação em saúde, de educação permanente e na formação acadêmica.

A educação em saúde está baseada no processo educativo referente a construção de conhecimentos quanto à situação de saúde da população, propondo uma maior autonomia do sujeito aos seus cuidados, estendendo-se a fatores sociais, contextos de vida e como estes podem influenciar na saúde (Brasil, 2013).

A Educação Permanente em Saúde envolve ações que proporcionam a problematização das atividades laborais dos trabalhadores de saúde, sendo atividades utilizadas para fins de modificação de práticas equivocadas de profissionais no ambiente de trabalho, a fim de melhorar a assistência em saúde (Brasil, 2013).

O desenvolvimento de TE também está intrínseco à Formação Acadêmica, em que o desenvolvimento da tecnologia seja com o propósito de inserção no ensino técnico, de graduação ou de pós-graduação.

\subsection{Tipos de fontes}

Serão considerados apenas artigos científicos produtos de pesquisas do tipo metodológicas, pois este tipo de pesquisa objetiva o desenvolvimento, a validação e avaliação de uma ferramenta (Polit \& Beck, 2011).

\subsection{Estratégias de pesquisa}

Foi realizada busca preliminar com a procura de scoping reviews semelhantes nas seguintes bases de dados: Open Science Framework (OSF), Joanna Briggs Institute COnNECT+, Database of Abstracts of Reviews of Effects (DARE), The Cochrane Library e International Prospective Register of Systematic Reviews (PROSPERO) e não foram identificados protocolos e revisões com temática semelhante.

Posteriormente, foi realizada busca inicial no portal Public Medical (PubMed) e na base de dados Biblioteca Virtual em Saúde (BVS) para identificar sinonímias dos termos de busca referentes à temática da pesquisa, sendo esta busca realizada a partir da combinação dos Descritores em Ciências da Saúde (DeCS) e dos Medical Subject Headings (MeSH) identificados para o mneumônico PCC desta revisão.

Dessa maneira, foi estabelecido a estratégia de busca a ser utilizada nas bases de dados (Quadro 1). Serão incluídos estudos de qualquer idioma e não será delimitado limite temporal. 
Quadro 1. Estratégia de busca construída com base em pesquisa exploratória nas bases de dados BVS e Pubmed.

Natal, RN, Brasil, 2021.

\begin{tabular}{|l|l|c|}
\hline $\begin{array}{l}\text { Base de dados: BVS } \\
\text { Data e hora da busca: 10/05/2021 - 14h }\end{array}$ & Palavras-chave identificadas \\
\hline Mneumônico & DeCS & Tecnologia Educativa, Materiais de Ensino \\
\hline P & Tecnologia Educacional & \multicolumn{1}{|c|}{ Estudos de Validação } \\
\hline C & Estudo de Validação & Área da Saúde \\
\hline C & Ciências da Saúde & Palavras-chave identificadas \\
\hline $\begin{array}{l}\text { Base de dados: Pubmed } \\
\text { Data e hora da busca: 10/05/2021 - 17h }\end{array}$ & Teaching Materials \\
\hline Mneumônico & MESH & Validation Studies, Validity, Validation, \\
\hline P & Educational Technology & Instrument Validation \\
\hline $\begin{array}{l}\text { C } \\
\text { MESH: (Educational technology) OR Teaching Materials) AND Validation study) OR Validation Studies) } \\
\text { OR validity) OR validation) OR instrument validation) AND health) OR Health Sciences }\end{array}$ \\
\hline $\begin{array}{l}\text { C } \\
\text { (tw:(Estudo de Validação) OR (tw:( Estudos de Validação)) AND (tw:( Ciências da Saúde)) OR (tw:(saúde)) }\end{array}$ \\
\hline $\begin{array}{l}\text { Estratégia Base Definida } \\
\text { Health }\end{array}$
\end{tabular}

Fonte: Autores (2021).

\subsection{Fontes de informações}

As bases de dados a serem pesquisadas incluem: PubMed, Cumulative Index to Nursing and Allied Health Literature (CINAHL), SCOPUS, WEB OF SCIENCE, American Psychological Association (APA PsycINFO), Educational Resources Information Centre (ERIC), Google Scholar e BVS.

Será utilizada a estratégia de busca de publicações de interesse nas referências dos estudos selecionados.

\subsection{Seleção dos estudos}

Após a seleção dos estudos, todos serão identificados, agrupados e inseridos no Software Rayyan, a fim de se remover os estudos duplicados. Os títulos e resumos serão analisados por dois revisores independentes para que possam avaliar os estudos de acordo com os critérios de inclusão e exclusão e os motivos de exclusão serão evidenciados na revisão. Quaisquer divergências interpretativas entre os dois revisores no processo de seleção dos estudos serão resolvidas por meio de uma discussão ou com o auxílio de um terceiro revisor. Serão excluídos: editoriais, artigos de opinião, ensaios teóricos e literatura não publicada em periódicos (literatura cinzenta).

\subsection{Extração de dados}

Os dados serão extraídos e incluídos na revisão de escopo por dois revisores de forma independente. As informações coletadas serão extraídas a partir de uma planilha construída no Microsoft Excel 2016 e incluirão detalhes específicos sobre a base de dados dos estudos: ano de publicação, local de origem do estudo e sua área de conhecimento. Informações sobre a população, conceito, contexto, referências, etapas utilizadas para a construção do estudo, abordagem metodológica e nível 
educacional em que a TE é utilizada serão evidenciadas por esta revisão (Quadro 2). Os autores realizarão uma pesquisa piloto com três estudos a fim de se familiarizar com a coleta dos dados. Divergências interpretativas entre os dois revisores, durante o processo de coleta de informações, serão resolvidas por meio de uma discussão ou com o auxílio de um terceiro revisor.

Quadro 2. Formulário de extração de dados.

\begin{tabular}{|l|l|}
\hline Variável & Padronização \\
\hline Base de dados & Conforme a origem durante o processo de seleção \\
\hline Ano de publicação & Ano em que o estudo foi publicado \\
\hline País & Local de origem do estudo \\
\hline Área da saúde & Campo de conhecimento principal da pesquisa \\
\hline Tipo de tecnologias educacionais & Detalhar quais os tipos de tecnologias utilizadas \\
\hline Tipo e abordagem de pesquisa & Detalhar tipo de pesquisa realizada \\
\hline População de estudo & Detalhar sujeitos participantes da pesquisa \\
\hline Tipo de validação & Detalhar a validação utilizada \\
\hline Referencial utilizado para validar a tecnologia & Detalhar o referencial utilizado para validação da tecnologia educacional \\
\hline Etapas do Processo de Validação & Detalhar o as etapas do processo de validação utilizado \\
\hline Nível educacional da publicação & $\begin{array}{l}\text { Detalhar quanto ao nível educacional da tecnologia educacional utilizada: } \\
\text { educação em saúde, formação acadêmica ou educação permanente }\end{array}$ \\
\hline
\end{tabular}

Fonte: Autores (2021).

\subsection{Apresentação dos dados}

Os dados extraídos serão sintetizados e apresentados de forma descritiva (n e \%), por meio de tabelas, gráficos e quadros, quando pertinente. Um resumo narrativo acompanhará as informações tabuladas e mapeadas a fim de descrever como os resultados se relacionam com o objetivo e a questão da revisão. Os resultados da pesquisa serão descrevidos na íntegra na revisão de escopo final e apresentados segundo o Preferred Reporting Items for Systematic Reviews and Meta-Analyses Extension for Scoping Reviews (PRISMA-ScR) para construção do artigo final (Tricco et al., 2018).

\section{Considerações Finais}

A criação deste protocolo possibilitará o desenvolvimento de uma revisão de escopo baseada nos princípios do manual for evidence synthesis de Joanna Briggs Institute e, a partir disto, uma obtenção de maiores conhecimentos acerca do processo de validação de tecnologias educacionais na área da saúde.

Para trabalhos futuros, sugere-se a construção de outros protocolos com metodologias consistentes que baseie a execução de novas revisões de literatura, sobretudo no âmbito da validação de tecnologias educacionais.

\section{Referências}

Alexandre, N. M. C., \& Coluci, M. Z. O. (2011). Validade de conteúdo nos processos de construção e adaptação de instrumentos de medidas. Ciencia e Saude Coletiva, 16(7), 3061-3068. 10.1590/S1413-81232011000800006.

Brasil. M. S. (2021). Guia de vigilância epidemiológica Emergência de saúde pública de Importância nacional pela Doença pelo coronavírus 2019 - covid19. https://www.conasems.org.br/wp-content/uploads/2021/03/Guia-de-vigilância-epidemiológica-da-covid_19_15.03_2021.pdf.

Brasil. M. S. (2013). Secretaria-Executiva. Secretaria de Gestão do Trabalho e da Educação na Saúde. Glossário temático: gestão do trabalho e da educação na saúde. http://bvsms.saude.gov.br/bvs/publicacoes/glossario_tematico_gestao_trabalho_educacao_saude_2ed.pdf.

Brasil. (2020). Portaria $n^{\circ} 343$, de 17 de março de 2020. Dispõe sobre a substituição das aulas presenciais por aulas em meios digitais enquanto durar a situação de pandemia do Novo Coronavírus - COVID-19. https://abmes.org.br/arquivos/legislacoes/Portaria-mec-343-2020-03-17.pdf. 
Carvalho, I. da S., Guedes, T. G., Bezerra, S. M. M. da S., Alves, F. A. P., Leal, L. P., \& Linhares, F. M. P. (2020). Educational technologies on sexually transmitted infections for incarcerated women. Revista Latino-Americana de Enfermagem, 28, e3392. 10.1590/1518-8345.4365.3392.

Harapan, H., Itoh, N., Yufika, A., Winardi, W., Keam, S., Te, H., Megawati, D., Hayati, Z., Wagner, A. L., \& Mudatsir, M. (2020). Coronavirus disease 2019 (COVID-19): A literature review. Journal of Infection and Public Health, 13(January), 667-673. 10.1016/j.jiph.2020.03.019.

Nascimento, C. C. L. do, Silva, B. V. da C., Oliveira, J. das G. C., Nascimento, M. de F. S. do, \& Ferreira, V. S. (2020). Tecnologia educacional para sala de imunização: elaboração de bundle sobre conservação de imunobiológicos. Research, Society and Development, 9(7), 1-11. 10.33448/rsd-v9i7.4032.

Nespoli, G. (2013). Os domínios da tecnologia educacional no campo da saúde. Interface: Communication, Health, Education, 17(47), 873-884. $10.1590 /$ S1414-32832013005000028.

Nietsche, E. A., Lima, M. G. R. de, Rodrigues, M. da G. S., Teixeira, J. A., Oliveira, B. N. B. de, Motta, C. A., Gribler, C. S., Gribler, V. M., Lucas, D. D. I., \& Farias, M. K. F. de. (2012). Innovative Technologies of Nursing Care. Revista de Enfermagem UFSM, 2(1), 182-189.

Paim, L. M. D., Nietsche, E. A., \& Lima, M. G. R. de. (2014). História da tecnologia e sua evolução na assistência e no contexto do cuidado de enfermagem. In Tecnologias cuidativo- educacionais: uma possibilidade para o empoderamento do(a) enfermeiro(a)? (1st ed., pp. 17-36). Moriá.

Pasquali, L. (2010). Instrumentação Psicológica: Fundamentos e práticas. Artmed.

PAZ, J. M. B., \& Teixeira, E. (2021). Educational technology about living better with cardiac insufficiency: validation study. Revista de Pesquisa Cuidado é Fundamental Online, 428-433. 10.9789/2175-5361.rpcfo.v13.7140.

Peters, M.D.J., Godfrey, C., McInerney, P., Munn, Z., Tricco, A.C., \& Khalil, H. Chapter 11: Scoping Reviews (2020a). In: AromatarisE, Munn Z (Editors). JBI Manual for Evidence Synthesis, JBI, 2020. https://synthesismanual.jbi.global/.

Polit, D. F., \& Beck, C. T. (2011). Fundamentos de pesquisa em enfermagem: avaliação de evidências para a prática de enfermagem. Artmed.

Rodrigues, I. L. A., Teixeira, L. F. S., \& Nogueira, L. M. V. (2019). Educational technology on oral contraception: construction shared with nurses reproductive assistance. Revista de Pesquisa Cuidado é Fundamental, 11(1), 53-58. 10.9789/2175-5361.2019.v11i1.53-58.

Santos, S. L. F. dos, Lopes, E. M., Passos, A. C. de B., \& Néri, E. D. R. (2020). Validação de cartilha sobre o uso de medicamentos durante a gestação com o público-alvo. Journal Health NPEPS, 5(1), 52-67. 10.30681/252610104508.

Teixeira, E., \& Mota, V. M. S. de S. (2011). Tecnologias educacionais em foco. Difusão Editora.

Teixeira, E. (2010). Tecnologias em Enfermagem: produções e tendências para a educação em saúde com a comunidade. Revista Eletrônica de Enfermagem, 12(4), 598-600. 10.5216/ree.v12i4.12470.

Tricco, A. C., Lillie, E., Zarin, W., O’Brien, K. K., Colquhoun, H., Levac, D., Moher, D., Peters, M. D. J., Horsley, T., Weeks, L., \& Hempel, S. (2018). PRISMA Extension for Scoping Reviews (PRISMA-ScR) : Checklist and Explanation. Annals of Internal Medicine., 467-473. 10.7326/M18-0850. 